The Complete Disc Manual for the BBC Microcomputer 


\section{Macmillan Microcomputer Books}

General Editor: Ian Birnbaum (Advisor for Microelectronics in Education, Hurnberside LEA)

Advanced Graphics with the Acorn Electron

Ian O. Angell and Brian J. Jones

Advanced Graphics with the BBC Model B Microcomputer

Ian O. Angell and Brian J. Jones

Interfacing the BBC Microcomputer

Brian Bannister and Michael Whitehead

Sorting Routines for Microcomputers

Angus Barber and Keith McKluckie

Assembly Language Programming for the Acorn Electron

Ian Birnbaum

Assembly Language Programming for the BBC Microcomputer (second edition)

Ian Birnbaum

Using Wordwise: An Instructional Guide

John Blower and Anne Cronin

Microchild: Learning through LOGO

Serafim Gascoigne

A Science Teacher's Companion to the BBC Microcomputer

Philip Hawthorne

Operating the BBC Microcomputer: A Concise Guide

Graham Leah

Beginning BASIC with the ZX Spectrum

Judith Miller

Using Sound and Speech on the BBC Microcomputer

Martin Phillips

A Beginner's Guide to Interfacing the BBC Microcomputer

Martin Phillips

File Handling on the BBC Microcomputer

Brian J. Townsend

Good BASIC Programming on the BBC Microcomputer

Margaret White

Also from Macmillan

Advanced Graphics with the IBM Personal Computer Ian O. Angell

Using Your Home Computer (Practical Projects for the Micro Owner)

Garth W. P. Davies

Micro-Maths

Keith Devlin

Using $C P / M$

Peter Gosling

Program Your Microcomputer in BASIC

Peter Gosling

Microprocessors and Microcomputers - Their Use and Programming

Eric Huggins

Programming in Z80 Assembly Language

Roger Hutty

Digital Techniques

Noel Morris

Microprocessor and Microcomputer Technology

Noel Morris

Computer Literacy: A Beginners' Guide

Vincent Walsh 


\title{
The Complete Disc Manual for the BBC Microcomputer
}

\author{
R. I. M. Sadek
}

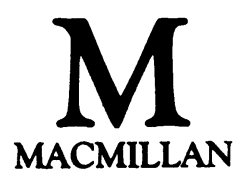


All rights reserved. No reproduction, copy or transmission of this publication may be made without written permission.

No paragraph of this publication may be reproduced, copied or transmitted save with written permission or in accordance with the provisions of the Copyright Act 1956 (as amended).

Any person who does any unauthorised act in relation to this publication may be liable to criminal prosecution and civil claims for damages.

First published 1986

Published by

MACMILLAN EDUCATION LTD

Houndmills, Basingstoke, Hampshire RG21 2XS

and London

Companies and representatives

throughout the world

Photoset by Parker Typesetting Service, Leicester

British Library Cataloguing in Publication Data

Sadek, R. I. M.

Complete disc manual for the BBC Microcomputer.

1. BBC Microcomputer 2. Floppy discs (Computer storage devices)

I. Title

001.64'42 QA76.8.B35

ISBN 978-0-333-40930-5 ISBN 978-1-349-08590-3 (eBook)

DOI $10.1007 / 978-1-349-08590-3$ 
For Karen 


\section{Contents}

1 Introduction 1

2 Basic Principles 3

5.25" floppy discs 4

Disc drives $\quad 6$

$\begin{array}{lr}\text { Tracks and sectors } & 10\end{array}$

$\begin{array}{ll}\text { Formatting } & 10\end{array}$

Double-density data storage $\quad 11$

$\begin{array}{ll}\text { Disc care } & 12\end{array}$

3 Files and Filenames $\quad 14$

$\begin{array}{ll}\text { The catalogue } & 15\end{array}$

Filenames $\quad 15$

Drive numbers 16

$\begin{array}{ll}\text { Directories } & 16\end{array}$

$\begin{array}{ll}\text { Library directories } & 18\end{array}$

$\begin{array}{ll}\text { Wildcards } & 19\end{array}$

4 Filing Commands $\quad 20$

$\begin{array}{lr}\text { Operating system commands } & 20\end{array}$

Language commands $\quad 22$

DFS commands $\quad 23$

$\begin{array}{ll}\text { Autoboot } & 28\end{array}$

$\star$ SPOOL and $\star$ EXEC $\quad 30$

$\star$ WIPE, $\star$ DELETE, $\star$ DESTROY $\quad 31$

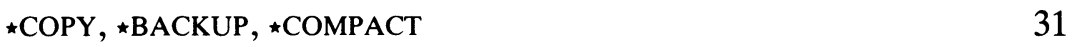

User-defined commands $\quad 33$

5 Errors $\quad 34$

DFS errors $\quad 34$

8271-instigated errors $\quad 36$

6 Cassette-to-Disc Transfer $\quad 38$

Memory usage $\quad 39$

Simple transfer and relocation techniques $\quad 41$

Memory usage revisited $\quad 44$

General principles $\quad 45$ 
$\begin{array}{ll}\text { Program types } & 47\end{array}$

$\begin{array}{ll}\text { Protected programs } & 50\end{array}$

7 BBC Basic Filing Commands and Functions 55

$\begin{array}{ll}\text { Opening and closing files } & 55\end{array}$

Filing system variables $\quad 57$

Data transfer: single byte $\quad 59$

Data transfer: strings, real numbers and integers $\quad 60$

8 Filing Techniques $\quad 63$

General principles $\quad 63$

$\begin{array}{ll}\text { File subdivision } & 66\end{array}$

$\begin{array}{ll}\text { Opening and closing files } & 68\end{array}$

$\begin{array}{ll}\text { Reading and writing data } & 72\end{array}$

$\begin{array}{ll}\text { File structures } & 76\end{array}$

Some more techniques $\quad 81$

9 Example Filing/Database Programs 83

10 Operating System Routines 106

$\begin{array}{ll}\text { OSWORD } & 107\end{array}$

Filing system calls $\quad 109$

11 Disc Layout 128

$\begin{array}{ll}\text { Catalogue sectors } & 128\end{array}$

Track layout and detailed structure $\quad 131$

12 The Intel 8271 Floppy Disc Controller 139

$\begin{array}{ll}\text { Registers } & 140\end{array}$

$\begin{array}{ll}\text { Commands } & 149\end{array}$

Data transfer commands $\quad 153$

Scan commands $\quad 155$

138271 Programming Examples 159

Disc formatter $\quad 159$

File recoverer $\quad 162$

$\begin{array}{ll}\text { Sector editor } & 164\end{array}$

14 DFS Memory Usage 179

Page 13, \&D00 179

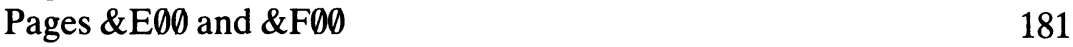

Page \&1000 181

Pages \&1100, and \&1200 to \&1600 181

Pages \&1700 and \&1800 182

Other locations relevant to the DFS 182

Appendices $\quad 195$

Bibliography 196

Index 197 